\title{
Assessment of Intravenous Drugs Admixtures Incompatibilities at Nekemte Referral Hospital, West Ethiopia
}

\author{
Balisa Mosisa*, Bokasa Negeri, Getu Bayisa, Edao Sado, Ginenus Fekadu, Mohammed Gebre, \\ Busha Gamachu, Sagaram Sudhakar
}

School of Pharmacy, Wollega University, Nekemte, Ethiopia

Email address:

balisamosis@yahoo.com (B. Mosisa)

${ }^{*}$ Corresponding author

To cite this article:

Balisa Mosisa, Bokasa Negeri, Getu Bayisa, Edao Sado, Ginenus Fekadu, Mohammed Gebre, Busha Gamachu, Sagaram Sudhakar. Assessment of Intravenous Drugs Admixtures Incompatibilities at Nekemte Referral Hospital, West Ethiopia. American Journal of Health Research. Vol. 8, No. 5, 2020, pp. 84-88. doi: 10.11648/j.ajhr.20200805.12

Received: September 28, 2020; Accepted: October 16, 2020; Published: October 23, 2020

\begin{abstract}
Intravenous admixtures are the preparations consisting of sterile drug products added to an IV fluid (s) for medication purposes. Especial concerns are required for such drugs since the reversal of toxicity and/or unwanted effects following incompatibilities are cumbersome to deal with. Concern drugs that may cause irritation or toxicity when given as a rapid direct IV injection are also prepared as IV admixtures. The ultimate goal of this assessment was to figure out the actual practices on the ground in IV medications administered in NRH with IV fluids emphasizing on incidence and prevalence of incompatibility between drugs themselves or with IV fluids. This study was a cross-sectional prospective observational study which investigated the potential incompatibilities associated with IV admixture, \& the incidence of IV drug administration errors using available evidence-based medicine sources at Nekemte Referral Hospital in West Ethiopia. The data was collected from patient medication Charts and prescription papers for patients treated within a period of 2 months. The study period was from February 2017 to April 2017 at the medical and pediatrics wards of NRH. About patients using IV admixtures were followed within this study period. The prevalence of identified incompatibility among IV admixtures ingredients was $28.94 \%$. The identified compatibility was only $25.43 \%$, while $38.59 \%$ were undocumented combinations, and $7.01 \%$ were variable. The most commonly encountered incompatible drug-solute combinations were Ringer lactate (61.8\%), Insulin + DNS (29.4) and Ceftriaxone + Phenytoin $+0.9 \% \mathrm{NaCl}(8.8 \%)$. Also, the incompatible drug-drug combinations detected in this study were Ceftriaxone+ heparin and clopidgrel+cimetidine combinations. Based on the findings of this study, the following can be concluded; there is a high prevalence of incompatibilities among IV admixtures. The types of incompatibilities were both drugsolute and drug-drug incompatibilities. Prescribers and administrators of IV admixtures were recommended to check plausibility before administration to prevent such incompatibilities which can compromise the treatment outcome of patients.
\end{abstract}

Keywords: IV Admixtures, Incompatibilities, Intravenous, Drugs, Nekemte Referral Hospital

\section{Introduction}

An IV admixture is a preparation of a pharmaceutical mixture of two or more drugs into a large bag or bottle of IV fluids mainly Normal Saline Solution $(0.9 \% \mathrm{NaCl})$ or Dextrose (5\%) alone or in combination under the direction of a doctor monitored by nurses and controlled by trained pharmacists. This is to be sure that no one is accidentally overdosed, or given the wrong medications [1].
Incompatibility is an undesirable reaction that occurs between the drug and the solute, container or another drug. The types of incompatibilities associated with intravenous administration are physical (Phase separation and precipitation due to a change of the relation between ionization and non-ionization, and solubility) and chemical (turbidity, precipitation, and color change due to drug degradation) [1].

The main causes of incompatibilities were mixing of drugs 
with inappropriate IV solutions (diluents); drug-drug incompatibilities when they are mixed together (within the same infusion lines and/or IV container administered one after the other, but within the same infusion line; drugs and materials of IV containers or medical devices, which can concern the nature of the material used and/or reactions at the inner surface $[1,2]$.

A Physical incompatibility (PI) occurs when a drug combination produces a visible change in the appearance of a solution [3]. It is also known as "Pharmaceutical Incompatibility or Visual Incompatibility" [4]. PIs are observed more easily and can be detected by changes in the appearance of admixtures such as a change in color, the formation of a precipitate, or the evolution of gas. This renders the solution unsafe for administration [5].

A Chemical incompatibility (CI) reflects the chemical degradation of 1 or more of the admixed drugs, resulting in toxicity or therapeutic inactivity [6]. The degradations are not always visible but the reaction of drugs or drugs in solutions which results in alterations in either integrity or potency of the drug [7]. Non-visible chemical incompatibility may be detected only by analytical methods [5].

IV admixture incompatibilities can lead to local redness, phlebitis, thrombophlebitis, thrombosis, local allergic reaction, minor organ dysfunction, respiratory difficulties, myocarditis, local embolus, systemic allergic reactions, organ failure, toxic shock, severe liver dysfunction, and multi organ failure $[3,6]$. Large amount of particulate matter in injections is considered as a potential life threatening health hazard [8].

Drug incompatibility reactions may not only generate particles in the infusion but also transform the drug into an inactive form and deleterious effects on the patients' prescribed drug regimen. Clinical effects of incompatibilities are hypoxia, impairment in the discharge of metabolic products, and finally tissue ischemia. These incompatibilities may therefore have the most detrimental effects in patients who suffer from preexisting microcirculatory disorders due to trauma, major surgery, and sepsis, etc [9]. The number of IV medications continues to expand and the need to administer different IV drug combinations is increasing day by day [10]. So, there is a need to know the situation in the specified study area for the following intervention.

\section{Methods}

\subsection{Study Setting}

Nekemte Town is located in the western part of Ethiopia. The study was conducted at Nekemte Referral Hospital, in Nekemte, which is about $331 \mathrm{~km}$ west of Addis Ababa. It is one of the main referral hospitals in western regions of the Nation.

\subsection{Study Design}

A prospective, quantitative, and cross-sectional survey designed to describe the current IV admixture practices at Nekemte Referral Hospital was used. Micromedex ${ }^{\circledR}$ and
LexiComponline drug reference software was used to screen patients for the presence of possible IV admixtures, and drugdrug interactions [11]. All drugs in a patient's medication profile were entered one by one into the software. The software display (s) all interacting combination (s) present in the medication profile. It also provides information about the mechanism and potential adverse outcomes of an interaction. Except for some modifications (additions) by the authors, definitions below are adapted from Micromedex ${ }^{\circledR}$.

Incompatibility is present when visible or electronicallydetermined precipitates, particulates, haziness, turbidity, color, or gas evolutions are detected. $10 \%$ or greater loss of intact drug within 24 hours is also considered evidence of incompatibility.

Drug-drug interaction (DDIs): Drug-drug interaction is the alteration of a drug's pharmacologic or clinical response by co-administered drug. Drug interaction can be of minor, moderate, and major type. Drug-drug interactions occur in the body when one drug disrupts the absorption, metabolism, action, or excretion of the other drug or when the pharmacologic effects of one drug increase or decrease the pharmacologic effects of the other drug.

\subsection{Data Collection and Analysis}

One trained pharmacy personnel collected data on the checklist prospectively by observing patients physically, using patients' prescriptions and medication records. The specific types of data necessary to measure compatibility status were recorded for each patient encounter and entered directly into the prepared compatibility checklist form. The number of patients for whom IV admixture was prescribed within the study period was. All data were first analyzed manually and then using Microsoft Excel 2007. In the statistical analysis, frequencies, averages/means, standard deviations, and percentages were obtained.

\subsection{Ethical Consideration}

Ethical approval was obtained from the Wollega University Research Vice President and Nekemte Referral Hospital. Then, written informed consents were collected from patients or care givers before data collections.

\section{Results and Discussion}

Samples of 144 patients who were using IV admixture were assessed prospectively in the in-patient ward of Nekemte Referral Hospital from February 5, 2017 to April 5, 2017. Data was collected and analyzed on the line of its objectives and presented below:

\subsection{Demographic Details and Characteristics of Patients}

A total of 232 patients were included in the study, of which $58.6 \%$ were males and $41.4 \%$ were females. The mean age was 35.5 years with the maximum number of patients being in the age group of 20 to 29 years. Concerning religion, majority $(49.1 \%)$ of the study patients were protestant followers. The 
ethnic composition has shown that $87.9 \%$ were Oromo. Concerning about marital status, $59.9 \%$ of them were married, $35.3 \%$ were single, and $4.7 \%$ were divorced. From a total of study subjects, majority of them $65.5 \%$ were primary school and $21.1 \%$ were no formal education (Table 1).

Table 1. Demographic profiles of the study subjects, NRH, Ethiopia, June, 2017

\begin{tabular}{|lll}
\hline $\begin{array}{l}\text { Demographics and } \\
\text { Characteristics Patients }\end{array}$ & $\begin{array}{l}\text { Percent } \\
\mathbf{( \% )}\end{array}$ \\
\hline Sex & Female & 41.4 \\
& Male & 58.6 \\
& $0-9$ & 10.3 \\
& $10-19$ & 12.9 \\
& $20-29$ & 18.5 \\
Age group & $30-39$ & 9.9 \\
& $40-49$ & 4.3 \\
& $50-59$ & 3.0 \\
& $60-69$ & 9.0 \\
Religion & $>70$ & 9.0 \\
& Protestant & 49.1 \\
& Orthodox & 25.4 \\
\hline Ethnicity & Muslim & 22 \\
& Catholic & 3.4 \\
\hline & Others & 3.0 \\
\hline & Oromo & 87.9 \\
\hline
\end{tabular}

\begin{tabular}{lll}
\hline $\begin{array}{l}\text { Demographics and } \\
\text { Characteristics Patients }\end{array}$ & Category & $\begin{array}{l}\text { Percent } \\
(\%)\end{array}$ \\
\hline \multirow{3}{*}{ Marital status } & Married & 59.9 \\
& Single & 35.3 \\
& Divorced & 4.7 \\
Educational level & Primary school & 65.5 \\
& No formal education & 21.1 \\
& Secondary school & 13.4 \\
\hline
\end{tabular}

Table 2. Types of diagnosed diseases for which IV admixture was prescribed in the study subjects, NRH, Ethiopia, June, 2017.

\begin{tabular}{ll}
\hline Type of diseases & Percentage \\
\hline Infectious diseases & 49.6 \\
Respiratory disorders & 20.7 \\
Cardio vascular diseases & 10.3 \\
Endocrine disorders & 6.0 \\
GI disorders & 3.9 \\
Kidney diseases & 3.4 \\
CNS disorders & 3.0 \\
Otherdiseases & 3.0 \\
Total & 100.0 \\
\hline
\end{tabular}

As depicted in table 2, about half $(49.6 \%)$ of all patients for which IV admixtures were prescribed were infectious diseases being followed by respiratory disorders (20.7\%). There is a considerable amount of contribution from cardiovascular diseases also $(10.3 \%)$.

\subsection{Compatibility Status of IV Admixtures}

Table 3. Compatibility status of IV admixtures administered to the study subjects in NRH, April 2017.

\begin{tabular}{|c|c|c|c|c|c|}
\hline \multirow{2}{*}{ Compatibility } & \multicolumn{2}{|c|}{ No. Of Drug - IV solution Combinations } & \multicolumn{2}{|c|}{ No. Of Drug - Drug Combinations } & \multirow{2}{*}{ Total (\%) } \\
\hline & Y-site & Mixture & Y-site & Mixture & \\
\hline Compatible & 8 & 0 & 18 & 3 & $29(25.43 \%)$ \\
\hline Incompatible & 2 & 0 & 27 & 4 & $33(28.94 \%)$ \\
\hline Variable & 1 & 0 & 7 & 0 & $8(7.01 \%)$ \\
\hline Undocumented & 5 & 0 & 36 & 3 & $44(38.59 \%)$ \\
\hline Total & 16 & 0 & 88 & 10 & $(100 \%)$ \\
\hline
\end{tabular}

The prevalence of incompatibility in this study, according to evidence-based medicine standards, was found to be $28.94 \%$. The clearly known degrees of compatibility from the combinations of administered IV admixtures was $25.43 \%$. Here there was $38.59 \%$ of undocumented IV admixtures, while $7.01 \%$ were variable (Table 4). A similar study was done by Gikic $\mathrm{M}$, et al. revealed that $86.3 \%$ of IV admixtures were compatible, $3.4 \%$ incompatible, while $10.3 \%$ were undocumented [12]. Again, Valia, et al. found that $71.6 \%$ of IV admixtures were compatible and 1.1\% incompatible, $27.3 \%$ were undocumented [13]. The most probable reason for increment for the prevalence of incompatible combinations in this study area maybe the absence of clinical pharmacist participation in the pharmaceutical care of patients during this study. We hadn't come across with practicing clinical pharmacist in this hospital for better patient treatment outcome on decision making concerning medications.

The identified types of incompatible drug-solute combinations were found to be Ceftriaxone + Ringer lactate (61.8\%), Insulin + DNS (29.4\%), and Phenytoin $+0.9 \%$
$\mathrm{NaCl}(8.8 \%)$ (Table 4$)$.

Giving ceftriaxone with Ringer lactate leads to binding of calcium which is found in Ringer lactate to ceftriaxone in the blood which in turn leads to the formation of precipitate in the kidney and lung. This has caused several neonatal deaths. According to the FDA, no patient of any age should receive simultaneous administration of ceftriaxone and any IV solution containing calcium through an IV tubing $\mathrm{Y}$-site [14].

Giving insulin with DNS leads to high blood sugar or fluid shifts in the body that lead to swelling fluid buildup in the lungs. Dextrose should be avoided if there is high blood sugar; if there are low potassium levels in the blood; if there is peripheral edema (swelling in the arms, feet, or legs); when fluids buildup in the lungs [15]. Also, if insulin is given along with DNS, it may be absorbed to the surface of IV infusion solutions containers (both glass and plastic containers) [14].

On the other hand, when Phenytoin is mixed with $0.9 \%$ $\mathrm{NaCl}$, visible crystals of Phenytoin get formed within 10-15 minutes, ultimately leading to physical incompatibility [16]. 
Table 4. Types of Incompatible drug-solute admixtures at NRH, April 2017.

\begin{tabular}{ll}
\hline Incompatible IV Admixture Type & Percentage \\
\hline Ceftriaxone + Ringer Lactate & 61.8 \\
Insulin + DNS & 29.4 \\
Phenytion $+0.9 \% \mathrm{NaCl}$ & 8.8 \\
Total & 100.0 \\
\hline
\end{tabular}

Table 5. Prevalence of drug-solute admixtures at NRH, April 2017.

\begin{tabular}{ll}
\hline IV Admixtures & Percentage \\
\hline Ceftriaxone $+0.9 \% \mathrm{NaCl}$ & 33.0 \\
Ceftriaxone $+40 \% \mathrm{Glucose}$ & 27.7 \\
Ampicillin $+0.9 \% \mathrm{NaCl}$ & 18.1 \\
Ceftriaxone $+\mathrm{DNS}$ & 10.6 \\
Cimetidine $+0.9 \% \mathrm{NaCl}$ & 6.4 \\
Atropine $+0.9 \% \mathrm{NaCl}$ & 2.1 \\
Ampicillin $+40 \% \mathrm{Glucose}$ & 2.1 \\
Total & 100.0 \\
\hline
\end{tabular}

The most prevalent drug-solute IV admixtures administered given for the study subjects were Ceftriaxone + $0.9 \% \mathrm{NaCl}(33 \%)$, Ceftriaxone $+40 \%$ Glucose $(27.7 \%)$, Ampicillin $+0.9 \% \mathrm{NaCl}(18.1 \%)$.

Table 6. Types of incompatible drug-drug IV admixtures at NRH, April 2017.

\begin{tabular}{lll}
\hline IV Admixtures & Frequency & Percent \\
\hline Ceftriaxone + Heparin & 1 & 50.0 \\
Clopidogrel+ Cimetidine & 1 & 50.0 \\
Total & 2 & 100.0 \\
\hline
\end{tabular}

The most prevalent drug-drug interacting combinations given in the form of IV admixtures were Ceftriaxone + Heparin, and Clopidogrel + Cimetidine. Drug interaction refers to the modification of response to one drug by another when they are administered simultaneously or in quick succession. The modification is mostly quantitative, i.e. the response is either increased or decreased in intensity, but sometimes it is qualitative, i.e. an abnormal or a different type of response is produced. When a combination of Ceftriaxone and Heparin will be given to patients, interactions are reported among people elsewhere [17]. Additive hypoprothrombinaemia and bleeding may be caused.

For the case of clopidogrel+ cimetidine, clopidogrel inhibits platelet aggregation and is indicated for the prevention of vascular ischemia associated with atherothrombotic events. Clopidogrel is a prodrug that is converted to its active form by drug-metabolizing enzymes CYP3A4 and 3A5, with contributions from CYP2C19, CYP2C9, and CYP1A2. For clopidogrel to work, enzymes in the liver (particularly CYP2C19) must convert (metabolize) the drug to its active form. Proton pump inhibitors such as omeprazole are inhibitors of CYP2C19. Pharmacokinetic interaction between clopidogrel and omeprazole has now been confirmed following two pharmacokinetic/pharmacodynamic interaction studies. The results from these studies show that co-administration of clopidogrel with omeprazole results in significantly reduced exposure to the active metabolite of clopidogrel. So, healthcare professionals are advised to avoid the concomitant use of clopidogrel with omeprazole and other CYP2C19 inhibitors such as esomeprazole, cimetidine, fluconazole, ketoconazole, voriconazole, etravirine, fluoxetine, and fluvoxamine [17]. Especially for patient groups who are poor metabolizers of the drug do not effectively convert Clopidogrel to its active form. In these patients, Clopidogrel has less effect on platelets, and therefore less ability to prevent heart attack, stroke, and cardiovascular death. It is estimated that 2 to $14 \%$ of the population are poor metabolizers; the rate varies based on racial background. This ineffectiveness on intended treatment may be enhanced beyond expected if the drug is given with cimetidine and similar drugs

The most simultaneously prescribed drugs in the study area were Ceftriaxone + Metronidazole (54.6\%), Furosemide + Ceftriaxone (13.4\%), Ceftriaxone + Ranitidine (9.3\%) and others $(22.8 \%)$.

Table 7. Types of undocumented drug-solute combinations at NRH, April 2017.

\begin{tabular}{lll}
\hline IV Admixtures & Frequency & Percent \\
\hline Diclofenac $+0.9 \% \mathrm{NaCl}$ & 7 & 87.5 \\
Diclofenac + Ringer Lactate & 1 & 12.5 \\
Total & 8 & 100.0 \\
\hline
\end{tabular}

From total provided drug-solute IV admixture, 8 cases $(7 \%)$ were undocumented whether there is incompatibility or not with enough shreds of evidence.

\section{Conclusion}

Based on the finding of this study, the most simultaneously prescribed drugs in the study area were Ceftriaxone + Metronidazole, Furosemide + Ceftriaxone, Ceftriaxone + Ranitidine respectively. The IV admixture practices in current study had shown significant deviation from the standards recommended either by giving incompatible combination or prescribing undocumented admixtures, whose compatibility status is not clear yet. There was a considerable prevalence of incompatibility cases of IV admixtures at NRH. The most prevalent types of incompatibilities occurred were Ceftriaxone + Ringer lactate, Insulin + DNS, and Phenytoin $+0.9 \% \mathrm{NaCl}$, Ceftriaxone + Heparin, and Clopidgrel+ Cimetidine.

\section{Recommendation}

Authors recommend all concerned body to incorporate clinical pharmacist in pharmaceutical care decision making; act according to national standard treatment guidelines; establishing drug and therapeutic committee; problem-based basic training in pharmacotherapy; drug information centers establishment; expansion of drug use evaluation system and preparation and dissemination of drug bulletins. Mixing of the solutions of parenteral drugs with knowledge of their compatibility is called for. Information regarding drug incompatibilities is well documented in computer databases, AHFS, and information leaflets published by pharmaceutical Companies. So, they were recommended to be consulted. 


\section{Abbreviations}

\author{
IV: Intravenous \\ NRH: Nekemte Referral Hospital \\ CY P450: Cytochrome p450 \\ DDI: Drug-drug interaction \\ $\mathrm{NaCl}$ : Sodium Chloride \\ DNS: Dextrose Normal Saline \\ NS: Normal Saline \\ RL: Ringer Lactate \\ FDA: Food and Drug Administration
}

\section{Competing Interest}

The authors declare that they have no competing interests. There is no conflict of interest in reviewing this paper.

\section{Acknowledgements}

We thank Wollega University Research Vice President Office and Nekemte Referral Hospital for funding, allowing and helping us to conduct this research. Also, we extend our gratitude to NRH staff for their good cooperation.

\section{References}

[1] Mohammad Albaz (2013). I. V. ADMIXTURE. https://prezi.com/rjzrxzawm_k/iv-admixture/. Retrieved on October 15, 2020.

[2] Small GA, Marshall I. Intravenous additives. In: Lawson DH Richards RME. Clinical Pharmacy and Hospital Drug Management. 1st edition. Britain: The University-Press, Cambridge. 1982; 239-60.

[3] John F. Sterile Products. In: Shargel L, Souney PK, Mutnick AH, Swanson LN. Comprehensive Pharmacy Review. 8th edition. New Delhi: Wolters Kluwer Pvt Ltd., 2010; 348-357.

[4] Hankins, Judy, et al., the Infusion Nurses Society Infusion Therapy in Clinical Practice. 2nd edition. Philadelphia: WB Saunders, 2001.

[5] Scott SA. The Prescription. In: Alfonso RG. Remington the
Science and Practice of Pharmacy. 20th edition, New York: Lippincott Williams and Wilkins. 2000; 1687-705.

[6] Peter Murney, et al To mix or not to mix-compatibilities of parenteral drug solutions: Aust. prescr. 2008 Aug; 31 (4): 91101.

[7] Phillips, Lynn, Manual of IV Therapeutics. 4th edition. Philadelphia: Saunders, 2005.

[8] Tuan Tran, Thomas C. Kupiec, et al., Quality-Control Analytical Methods: Particulate Matter in Injections: What is it and what are the Concerns: International Journal of Pharmaceutical Compounding., 2006 May/June; 10 (3): 202204.

[9] K. V. Ramanath, Hymavathi, et al., Assessment of intravenous admixtures in hospitalized patients of a rural tertiary care teaching hospital: American journal of pharma tech research, 2012; 2 (4): 534-543.

[10] British National Formulary 57th edition March., 2009.

[11] Micromedex ${ }^{\circledR}$ HealthCare Series (internet database) Version 2.0. Greenwood Village, Colorado: Thomson Healthcare Inc; 2013. [http://www.micromedexsolutions.com]; https://online.lexi.com.

[12] M. Giki, E. R. Di Paolo, et al. Evaluation of physicochemical incompatibilities during parenteral drug administration in a pediatric intensive care unit: Pharm World Science., 2002; 22 (3): 88-91.

[13] Valia HD, Pierre V, Laurent G, Rene C, Andre P. Evaluation of intravenous drugs compatibilities in an adult ICU. $35^{\text {th }}$ European symposium on Clinical Pharmacy, 2006.

[14] Drug Interactions Checker - For Drugs, Food \& Alcohol. https:www.drugs.com_interraction_.

[15] Modern Weng, D. O. and Rachel Nall, MSN, CRNA (2018). Dextrose. Healthline. Retrieved on October 15, 2020.

[16] Ceftriaxone and Heparin drug interactions. Ehealthme. Realworld drug outcomes. http://www.ehealthme.com/druginteraction/ceftriaxone/heparin/.

[17] Medsafe. 2010. Clopidogrel and proton pump inhibitors possible interaction. Prescriber Update 31 (1): 2. Retrieved on October 15, 2020. 